\title{
Influence of strain on the kinetics of phase transitions in solids
}

\author{
Efim A. Brener, ${ }^{1}$ V. I. Marchenko, ${ }^{2}$ and R. Spatschek ${ }^{1}$ \\ ${ }^{1}$ Institut für Festkörperforschung, Forschungszentrum Jülich, D-52425 Jülich, Germany \\ ${ }^{2}$ P.L. Kapitza Institute for Physical Problems, RAS 119334, Kosygina 2, Moscow, Russia \\ (Received 31 August 2006; revised manuscript received 8 January 2007; published 16 April 2007)
}

\begin{abstract}
We consider a sharp interface kinetic model of phase transitions accompanied by elastic strain, together with its phase-field realization. Quantitative results for the steady-state growth of a new phase in a strip geometry are obtained, and different pattern formation processes in this system are investigated.
\end{abstract}

DOI: 10.1103/PhysRevE.75.041604

PACS number(s): 81.10. $-\mathrm{h}, 64.70 . \mathrm{Kb}, 46.15 .-\mathrm{x}, 47.54 .-\mathrm{r}$

\section{INTRODUCTION}

Many magnetic, superconducting, and structural phase transitions in solids are accompanied by small lattice distortions which lead to the presence of elastic deformations. For each phase transition a characteristic deformation can be assigned-i.e., the deformation acquired by the new phase relative to the initial phase in the absence of external forces. In some cases, these effects are of minor influence and can be ignored but, nevertheless, for many applications the elastic strain causes qualitatively new and observable effects. The influence on the thermodynamics of transitions between different phases has been thoroughly discussed in the literature (for a review see $[1,2]$, and, for more recent developments, e.g., [3] and references therein).

One of the well-known consequences is a thermodynamic elastic hysteresis-i.e., the splitting of the phase equilibrium point into two points: the points of the direct and inverse transition. It is mainly due to the coherency at the interphase boundary, meaning that the lattice layers remain continuous through the boundary. Correspondingly, the hysteresis disappears without interface coherence [4]. Despite the general understanding, some features of such transformations are still unclear, or at least under debate. For example, a distinctive two-phase equilibrium is established in the system within a certain temperature interval. The nature of this phenomenon is difficult to understand from the standpoint of ordinary thermodynamic equilibrium concepts: during the process of martensitic transformations the composition of the phases does not change, and thus in such systems only one phase can be stable at a given temperature.

However, the systematic theoretical study of the growth kinetics of such phase transitions accompanied by a lattice strain is much less advanced. In real systems the influence of elastic strain is often screened by many other effects-for example, by inhomogeneous compositions and temperature distributions, the Mullins-Sekerka instability, crystal anisotropy, polycrystalline structures, etc. Here, molecular dynamics simulations [5] and phase-field modeling of such complicated systems (see, for example, [6] and references therein) can lead to qualitative descriptions of the kinetics of phase transitions in solids. Recently, the growth of inclusions under elastic and thermal influence was discussed in [7], but there only spherical inclusions are considered.

In order to propel further progress in this field, the purpose of the current paper is threefold.
First, we want to develop a "minimum" kinetic model, from which even quantitative results concerning the influence of strain effects can be obtained. It differs from earlier descriptions in the sense that explicit equations of motion for sharp interfaces are set up and finally solved numerically. We believe that in the current stage of understanding it is very important to develop and understand relatively simple models which contain strain effects as the central ingredient. These models can later be supplemented by additional physical effects to approach step by step fully realistic and experimentally relevant behaviors.

Second, it is quite remarkable that already such a simple model leads to peculiar interfacial pattern formation processes due to strain effects.

Third, we use specific geometrical setups to demonstrate the applicability of the present description for the kinetics of solid-solid transitions. A strip geometry turns out to particularly interesting, because here a steady-state growth regime exists. We are not aware of any experimental results in this direction and hope to stimulate investigations which can be compared to the quantitative predictions derived here. Nevertheless, the model is of course not restricted to such cases and we use them mainly to demonstrate its applicability.

The paper is organized as follows: In the next section, we start with the investigations of solid-solid transitions, where the phases differ only in the size of the elementary cell, but have the same structure. After discussing the thermodynamics of this process, we study the steady-state growth in a strip, based on a sharp interface description in the spirit of first-order phase transitions. This leads to a moving boundary problem which is solved by phase-field methods as described in Sec. III. Phase transformations without change of size of the unit cell, which is only sheared, are investigated in Sec. IV.

\section{DILATATIONAL MISMATCH MODEL}

We start from the thermodynamical description of our model. The free-energy density of an initial phase is

$$
F_{1}=F_{1}^{0}+\frac{1}{2} \lambda u_{i i}^{2}+\mu u_{i k}^{2},
$$

where $F_{1}^{0}$ is the free-energy density without elastic effects, $u_{i k}$ are the components of the strain tensor, and $\lambda$ and $\mu$ are the elastic moduli of isotropic linear elasticity. The freeenergy density of a new phase is 


$$
F_{2}=F_{2}^{0}+\frac{1}{2} \lambda\left(u_{i i}-u_{i i}^{0}\right)^{2}+\mu\left(u_{i k}-u_{i k}^{0}\right)^{2}
$$

with $u_{i k}^{0}$ being a characteristic lattice strain assigned to the phase transition.

Let us consider the simplest case $u_{i k}^{0}=\varepsilon \delta_{i k}$, at first, where the bond lengths of the new phase are uniformly longer or shorter in all directions in comparison to the original phase; a specific example is the ferrite-to-austenite transformation in a ternary Fe-C 0.463 at. \%-Mn 0.496 at. \% alloy [6]. We assume that the elastic effects are small, $\varepsilon \ll 1$, and neglect the difference between the elastic coefficients in the two phases. Since in our description the reference state for both phases is the undeformed initial phase [see Eqs. (1) and (2)], the coherency condition at the interface reads $\mathbf{u}^{(1)}=\mathbf{u}^{(2)}$, where $\mathbf{u}$ is the displacement vector. The superscripts (1) and (2) refer to the initial and newly created phases respectively. Mechanical equilibrium at the interface demands $\sigma_{n n}^{(1)}=\sigma_{n n}^{(2)}$ and $\sigma_{n \tau}^{(1)}=\sigma_{n \tau}^{(2)}$. Here, the components of the stress tensor are given by

$$
\sigma_{i k}=\frac{1}{2}\left(\frac{\partial F}{\partial u_{i k}}+\frac{\partial F}{\partial u_{k i}}\right) ;
$$

the indices $n$ and $\tau$ denote the normal and tangential directions with respect to the interface. The condition of phase equilibrium requires the continuity of a new potential

$$
\tilde{F}=F-\sigma_{n n} u_{n n}-2 \sigma_{n \tau} u_{n \tau}
$$

across the flat interface [8], which takes into account the coherency constraint. In the general case of curved interfaces also the surface energy $\gamma$ has to be incorporated and the phase equilibrium condition for each interface point reads in the case of isotropic surface energy

$$
\widetilde{F}_{1}-\widetilde{F}_{2}-\gamma \mathcal{K}=0
$$

where $\mathcal{K}$ is the local curvature of the interface.

A critical nucleus of the new phase inside an unbounded initial phase can exist only if

$$
F_{1}^{0}-F_{2}^{0}>E \varepsilon^{2} /(1-\nu),
$$

where $E$ and $\nu$ are Young's modulus and Poisson ratio, respectively. This condition corresponds to the elastic hysteresis mentioned above. It can be obtained using the analogy of this elastic problem to the problem of thermal expansion for a given temperature field [9].

We discuss a simple strip configuration which allows the steady-state growth of an elastic "finger" consisting of the new phase (see Fig. 1), as a possible setup for experimental investigations. The unstrained elastic strip of width $L$ is attached to fixed grips at the upper and lower boundaries (u $=0$ there) and initially composed of the reference phase. We discuss a two-dimensional elastic problem using plane strain conditions $\left(u_{z}=0\right)$. Also, we assume the complete wetting of the walls by the initial phase. Thus, the new phase avoids a direct touching of the walls (see Fig. 1). Far ahead of the propagating finger the initial phase remains unstrained. In contrast, far behind the tip a phase coexistence is possible within a certain parameter interval near the transition tem-
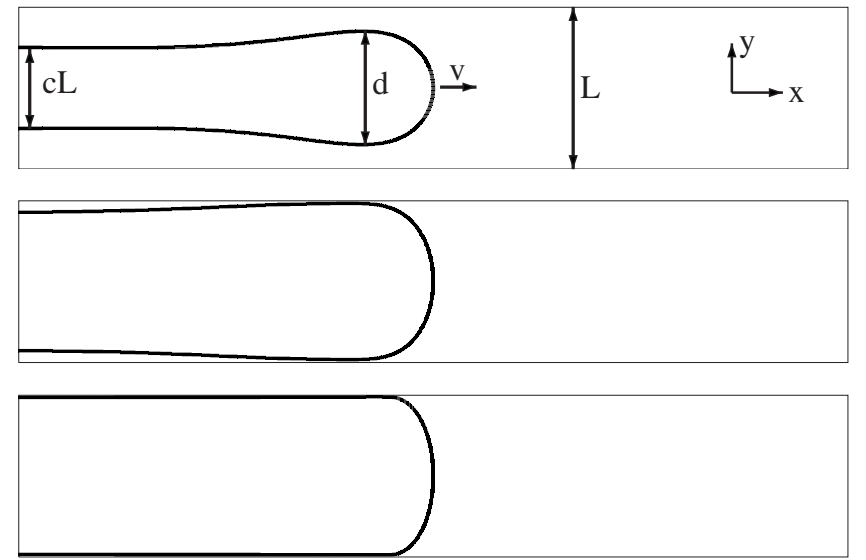

FIG. 1. Shapes of propagating fingers calculated for three values of the driving force; see Eq. (8). Top: $\Delta=0.5<\Delta_{t}$. Center: $\Delta$ $=0.85>\Delta_{t}$. Bottom: $\Delta=1.05$. The Poisson ratio is $\nu=1 / 3$ and $L / L^{*}=10$.

perature, which is due to elastic effects. In this region the only nonvanishing component of the displacement vector is $u_{y}$. The strain tensors are constant in both phases, and their nonzero components are connected to each other by the relation $(1-c) u_{y y}^{(1)}+c u_{y y}^{(2)}=0$, in order to fulfill the conditions $u_{y}=0$ on the walls and $u_{y}^{(1)}=u_{y}^{(2)}$ at the interface. Here, $c$ is the volume fraction of the new phase. Then, the mechanical equilibrium condition gives

$$
u_{y y}^{(1)}=-\frac{1+\nu}{1-\nu} c \varepsilon, \quad u_{y y}^{(2)}=\frac{1+\nu}{1-\nu}(1-c) \varepsilon .
$$

Taking into account the phase equilibrium condition, Eq. (5), we find the volume fraction of the new phase,

$$
c=\Delta=\frac{1-2 \nu}{1+\nu}\left[\frac{1-\nu}{E \varepsilon^{2}}\left(F_{1}^{0}-F_{2}^{0}\right)-1\right],
$$

which defines a dimensionless driving force $\Delta$ for this process. Then the parameter range for coexistence is $0<\Delta<1$. We note that the lower bound of this interval corresponds to the nucleation condition (6). The total free-energy gain per unit area in the $x z$ plane of this two-phase configuration compared to the unstrained initial phase is

$$
\Delta \mathcal{F}=L\left[F_{1}^{0}-c F_{2}-(1-c) F_{1}\right]-2 \gamma .
$$

Finally, using Eq. (8) we find

$$
\Delta \mathcal{F}=2 \gamma\left(\frac{\Delta^{2}}{\Delta_{L}^{2}}-1\right),
$$

where

$$
\Delta_{L}^{2}=\frac{L^{*}}{L}, \quad L^{*}=\frac{4(1-2 \nu)(1-\nu) \gamma}{(1+\nu) E \varepsilon^{2}} .
$$

The finger grows if $\Delta \mathcal{F}>0$ or, equivalently, if $\Delta>\Delta_{L}$. This condition corresponds to the Griffith point in fracture mechanics.

Two remarks are in order. First, we obtain the two-phase structure only because we take into account the elastic ef- 
fects and use the fixed-volume boundary condition. For stress-free boundaries, we would obtain $c=1$ for any driving force $F_{1}^{0}-F_{2}^{0}$ above the threshold (6) and $c=0$ below the threshold. Second, the value of the driving force for the transition can be controlled not only by temperature, but also by external strain. In particular, if the strip of width $L$ is stretched by $\delta L$, the homogeneous term $\delta L / L$ has to be added to the strain $u_{y y}$. This leads only to a renormalization of the driving force,

$$
F_{1}^{0}-F_{2}^{0} \rightarrow F_{1}^{0}-F_{2}^{0}+\frac{E \varepsilon \delta L}{L(1-2 \nu)} .
$$

Following our general aim to develop a minimum kinetic model, we assume that the growth is controlled only by interface kinetics. Then, the local equation of motion of the interface reads

$$
v_{n}=\kappa\left(\widetilde{F}_{1}-\widetilde{F}_{2}-\gamma \mathcal{K}\right),
$$

where $v_{n}$ is the normal velocity and $\kappa$ a kinetic coefficient.

Conservation of energy requires that the excess $\Delta \mathcal{F}$ be compensated for by dissipation at the interface. Integration of Eq. (13) for steady-state growth leads to a relation between the growth velocity and the driving force for the process,

$$
\frac{v}{v_{0} L} \int n_{x}^{2} d s=\Delta^{2}-\Delta_{L}^{2},
$$

where $v$ is the steady-state velocity of the finger and

$$
v_{0}=2 \kappa \gamma / L^{*}=\frac{\kappa(1+\nu) E \varepsilon^{2}}{2(1-2 \nu)(1-\nu)}
$$

is the characteristic velocity scale for this system; $n_{x}$ is the projection of the interface normal on the growth direction $x$ and the integration is performed along the interface. The dimensionless quantity $\int n_{x}^{2} d s / L$ is a complicated function of the parameters $\Delta, \Delta_{L}$, and $\nu$; in the case of dynamical elasticity, it also depends on the ratio $v_{0} / c_{s}$ where $c_{s}$ is the shear wave speed. Near the equilibrium point $\Delta=\Delta_{L}$, the growth velocity behaves as

$$
v / v_{0} \propto \Delta-\Delta_{L} .
$$

These results are valid for the dimensionless driving force $\Delta<1$. For $\Delta>1$ the fraction of the second phase becomes unity and Eq. (14) should be replaced by

$$
\frac{v}{v_{0} L} \int n_{x}^{2} d s=2 \Delta-1-\Delta_{L}^{2} .
$$

\section{PHASE-FIELD MODELING}

In order to obtain quantitative results for this problem we use a phase-field code as numerical tool, together with elastodynamics to describe phase transformations accompanied by stress, which we developed recently [10]. Let $\phi$ denote the phase field with values $\phi=1$ for the initial phase and $\phi$ $=0$ for the new phase. The free-energy density contribution is

$$
F=F_{1} h(\phi)+F_{2}[1-h(\phi)],
$$

where the switching function $h(\phi)=\phi^{2}(3-2 \phi)$ interpolates between the phases. The gradient energy is

$$
F_{s}(\phi)=\frac{3 \gamma \xi}{2}(\nabla \phi)^{2},
$$

with the interface width $\xi$. Finally,

$$
F_{d w}=\frac{6 \gamma}{\xi} \phi^{2}(1-\phi)^{2}
$$

is the double-well potential. Thus, the total free-energy functional is

$$
\widetilde{\mathcal{F}}=\int d V\left(F+F_{s}+F_{d w}\right) .
$$

The elastodynamic equations are derived from the free energy by variation with respect to the displacements $u_{i}$,

$$
\rho \ddot{u}_{i}=-\frac{\delta \tilde{\mathcal{F}}}{\delta u_{i}},
$$

where $\rho$ is the mass density. The dissipative phase field dynamics follows from

$$
\frac{\partial \phi}{\partial t}=-\frac{\kappa}{3 \xi} \frac{\delta \tilde{\mathcal{F}}}{\delta \phi} .
$$

These equations lead in the limit $\xi \rightarrow 0$ to the sharp interface description above. For the case of static elasticity, this was proven in [11].

For the numerical realization, we employ explicit representations of both the elastodynamic equations and the phase-field dynamics. We shift the grid horizontally in order to keep the propagating tip always in the center of the strip; this allows us to study steady-state growth in moderately large systems. The intrinsic length scale $L^{*}$ is chosen to be larger than the phase-field interface width, $L^{*}=8 \xi$ and $\xi$ $=5 \Delta x$, where $\Delta x$ is the numerical lattice unit. We have performed calculations for the Poisson ratio $\nu=1 / 3$ and for two values of the dimensionless strip width $L / L^{*}=10$ and $L / L^{*}$ $=20$ or, equivalently, for two values of the parameter $\Delta_{L}$ $\approx 0.32$ and $\Delta_{L} \approx 0.22$. All simulations are conducted on the parallel computer JUBL operated at the Research Center Jülich.

First of all, we have checked that the asymptotic conditions in the two-phase region far behind the tip, Eqs. (7) and (8), are reproduced by our numerics with high precision. Though the appearance of the Asaro-Tiller-Grinfeld instability $[12,13]$ might be naively expected in the tail region because of the presence of nonhydrostatic stresses, this effect is never observed in the simulations. In the limit $L \rightarrow \infty$, we have checked analytically that the system is stable. We have also confirmed the predicted renormalization of the driving force (12) due to an external strain $\delta L / L$. Next, we have investigated the properties of growing elastic fingers for different driving forces $F_{1}^{0}-F_{2}^{0}$. After a transient regime the finger always reaches the steady-state configuration. Characteristic shapes of the stationary fingers are presented in Fig. 


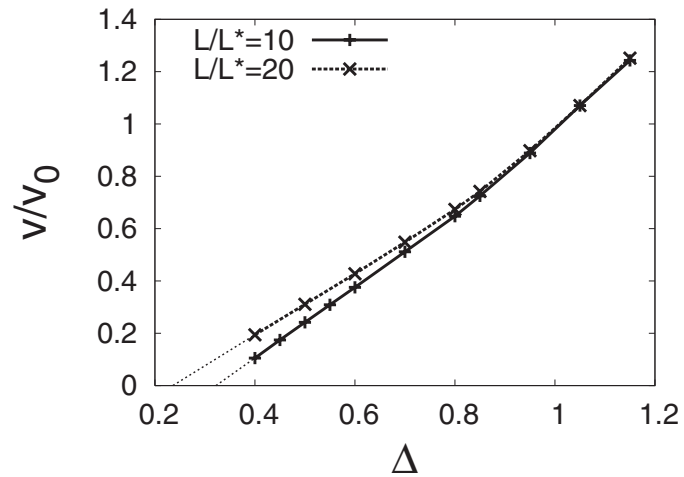

FIG. 2. The dimensionless growth velocity $v / v_{0}$ as a function of the dimensionless driving force $\Delta$.

1. The finger selects a symmetrical shape even if the initial configuration was vertically off centered; i.e., the symmetrical configuration is stable. We note the existence of the "bubble" in the tip region (top panel of Fig. 1). The bubble touches the walls at a specific value $\Delta=\Delta_{t}<1$. With further increase of the driving force the touching region smoothly increases and diverges at $\Delta=1$ (see middle and bottom panels of Fig. 1).

The dependence of the dimensionless steady-state growth velocity $v / v_{0}$ on the dimensionless driving force $\Delta$ is presented in Fig. 2. The velocity rises from zero at $\Delta=\Delta_{L}$ with increasing driving force. It turns out that the dependence is almost linear in a wide range, $\Delta_{L}<\Delta<\Delta_{t}$. This is a slightly unexpected result, because the linear behavior is anticipated to be valid only in the close vicinity of the equilibrium point $\Delta=\Delta_{L}$. At both critical points $\Delta=\Delta_{t}$ and $\Delta=1$, the growth velocity is a continuous function of the driving force.

All the results presented so far have been obtained for the characteristic velocity scale $v_{0}$ being much smaller than the shear-wave speed-i.e., in the limit of static elasticity. We have performed additional runs with higher characteristic velocities such that $v / c_{s} \approx 1 / 2$, where dynamical effects are relevant. For the same driving force, the dimensionless velocity $v / v_{0}$ decreases compared to the static elasticity limit and the size of the bubble grows in order to reach the same dissipation according to Eq. (14).

\section{TRANSITIONS WITH SHEAR STRAIN}

A simple type of transition involving shear strain can occur in hexagonal crystals. For the transitions lowering the symmetry from $C_{6}$ to $C_{2}$ the shear strain in a basic plane appears (see, e.g., [1]). This is the case for example in hexagonal-orthorhombic transitions in ferroelastics (see [14] and references therein). For simplicity we neglect all other possible strains with higher (axial) symmetry. We assume that the crystal is attached to two parallel walls as before. Let the principal axis $C_{6}$ be oriented in $z$ direction. By proper choice of the crystal orientation around the main axis in the initial phase, we obtain the new phase in three possible states due to the original hexagonal symmetry. Then the nonvanishing components of the strain tensor $u_{i k}^{0}$ are $u_{x x}^{0}=-u_{y y}^{0}$ $=\varepsilon \cos 2 \theta$ and $u_{x y}^{0}=\varepsilon \sin 2 \theta$, where the angle $\theta$ is $\theta$

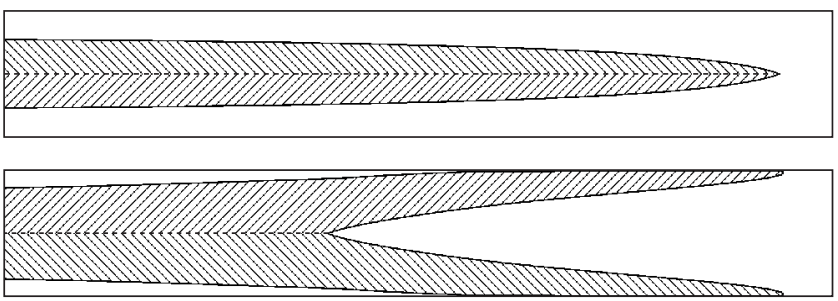

FIG. 3. Growth of bicrystal patterns for $L / L^{*}=5$. The shading illustrates the orientation of the shear strain $u_{x y}$. Forward slashes correspond to $\theta=-2 \pi / 3$, backslashes to $\theta=2 \pi / 3$. The strip lengths used in the simulations are much larger than in the sections shown here; far away in the tail region, both shapes have concentrations $c=\Delta=0.6$. The growth velocities are $v / v_{0}=1.14$ (top) and $v / v_{0}$ $=0.48$ (bottom).

$=0, \pm 2 \pi / 3$. Because the elasticity of hexagonal crystals is axisymmetric in harmonic approximation and $u_{i z}^{0}=u_{i z}=0$ for the discussed problem, we can use Eqs. (1) and (2) for the free-energy densities of the two phases (see, e.g., [9]). The moduli of the effective isotropic elasticity, $\lambda$ and $\mu$, can be expressed in terms of the elastic constants of the original hexagonal crystal.

A straightforward analysis of the stress state far behind the tip similar to Eqs. (7)-(9) shows that among the possible configurations of new phases the energetically most favorable scenario are bicrystals, $\theta= \pm 2 \pi / 3$, as presented in Fig. 3. In the asymptotic tail region $u_{x y}^{(1)}=0$ and $u_{x y}^{(2)}=\mp \varepsilon \sqrt{3} / 2$, where different signs correspond to different domains of the bicrystals. The distribution of the strain component $u_{y y}$ and the fraction of the new phase $c$ can be readily found in the same way as before. For example, Eq. (8) should be replaced by

$$
c=\Delta=\frac{1}{1-2 \nu}\left[\frac{4\left(1-\nu^{2}\right)}{E \varepsilon^{2}}\left(F_{1}^{0}-F_{2}^{0}\right)-\frac{1}{2}\right] .
$$

The presence of the twin boundary with interfacial energy $\gamma_{b}$ requires also a modification of the characteristic length and velocity scales,

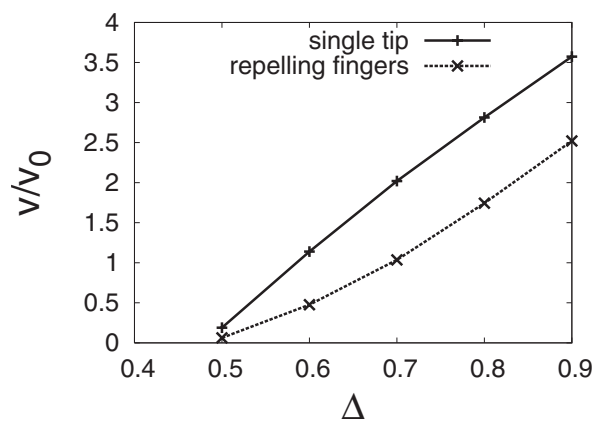

FIG. 4. Growth velocity of the different bicrystals for $L / L^{*}=5$. A slight discrepancy from the equilibrium point $\Delta_{L}$ is due to the accumulation of elastic energy in the transition region of the phase field, which leads to a renormalization of the surface energy. We checked numerically that this effect is suppressed in the sharp interface limit, as expected. 


$$
L^{*}=\frac{8\left(1-\nu^{2}\right)\left(2 \gamma+\gamma_{b}\right)}{(1-2 \nu) E \varepsilon^{2}}, \quad v_{0}=\frac{\kappa\left(2 \gamma+\gamma_{b}\right)}{L^{*}},
$$

compared to Eqs. (11) and (15); moreover, it leads to the existence of a triple junction in the tip region.

For a numerical phase field study of these twin structures we immediately take into account the symmetry of the appearing patterns and describe only either the upper or lower halves of the strip. At the symmetry plane the boundary conditions are $u_{y}=0, \sigma_{x y}=0$, and, for the specific case $\gamma_{b} \ll \gamma$ considered here, $\partial \phi / \partial y=0$. This avoids more complicated multiphase descriptions which are in principle capable of describing the three different phases. Although both patterns in Fig. 3 are energetically equivalent far away from the tip, symmetry is broken by the choice of the propagation direction. For growth to the right, the orientation $\theta=2 \pi / 3$ in the upper and $\theta=-2 \pi / 3$ in the lower half, leading to propagation with a single tip (upper panel of Fig. 3), is superior to the opposite case with repelling fingers (lower panel), as the growth velocity is higher; see Fig. 4.

\section{SUMMARY}

We developed a simple sharp-interface kinetic model of strain influenced phase transitions in solids. To solve the arising moving boundary problem we designed a suitable phasefield realization. We obtained quantitative results for the steady-state growth of an elastic finger in a strip geometry and discussed the peculiar behavior of different pattern formation processes in this system. The influence of additional fields-e.g., composition and temperature-will be the subject of future investigations.

\section{ACKNOWLEDGMENTS}

This work has been supported in part by the Deutsche Forschungsgemeinschaft under Grant No. SSP 1120, the German-Israeli Foundation, and by CRDF Grant No. RUE11625-MO-06. V.I.M. thanks Forschungszentrum Jülich for hospitality.
[1] A. L. Roitburd, Sov. Phys. Usp. 17, 326 (1974).

[2] A. G. Khachaturyan, Theory of Structural Transformation in Solids (Wiley, New York, 1983).

[3] L. Delaey, in Phase Transformations in Materials, edited by G. Kostrorz (Wiley-VCH, Weinheim, 2001), p. 585.

[4] E. A. Brener and V. I. Marchenko, JETP Lett. 56, 368 (1992).

[5] P. S. Branicio, R. K. Kalia, A. Nakano, and P. Vashishta, Phys. Rev. Lett. 96, 065502 (2006).

[6] I. Steinbach and M. Apel, Physica D 217, 153 (2006).

[7] J. Slutsker, K. Thornton, A. L. Roytburd, J. A. Warren, and G. B. McFadden, Phys. Rev. B 74, 014103 (2006).
[8] I. A. Privorotskii, Sov. Phys. JETP 33, 825 (1971).

[9] L. D. Landau and E. M. Lifshitz, Theory of Elasticity (Pergamon Press, Oxford, 1987).

[10] R. Spatschek, M. Hartmann, E. Brener, H. Müller-Krumbhaar, and K. Kassner, Phys. Rev. Lett. 96, 015502 (2006).

[11] K. Kassner, C. Misbah, J. Müller, J. Kappey, and P. Kohlert, Phys. Rev. E 63, 036117 (2001).

[12] R. J. Asaro and W. A. Tiller, Metall. Trans. 3, 1789 (1972).

[13] M. A. Grinfeld, Sov. Phys. Dokl. 31, 831 (1986).

[14] S. H. Curnoe and A. E. Jacobs, Phys. Rev. B 63, 094110 (2001). 